\title{
Prevalência de ansiedade relacionada ao tratamento odontológico em adolescentes: revisão integrativa da literatura
}

\author{
Prevalence of dental anxiety in adolescents: integrative review \\ Prevalencia de ansiedad relacionada al tratamiento odontológico en adolescentes: revisión integrativa de la literatura \\ Trícia Murielly Andrade de Souza MAYER ${ }^{1}$ \\ Patrícia Diletieri ASSIS ${ }^{2}$ \\ Michele Gomes do NASCIMENTO ${ }^{1}$ \\ Kamila Azoubel BARRETO ${ }^{1}$ \\ Carolina Maria Maciel Pessoa da SILVA² \\ Viviane COLARES ${ }^{3}$ \\ ${ }^{I}$ Doutoranda em Odontopediatria, Faculdade de Odontologia de Pernambuco, Universidade de Pernambuco (FOP/UPE), 54756-220, Camaragibe-PE, Brasil \\ ${ }^{2}$ Mestranda em Odontopediatria, Faculdade de Odontologia de Pernambuco, Universidade de Pernambuco (FOP/UPE), 54756-220, Camaragibe, PE, Brasil \\ ${ }^{3}$ Professora Associada de Odontopediatria, Faculdade de Odontologia de Pernambuco, Universidade de Pernambuco (FOP/UPE), 54756-220, Camaragibe, PE, Brasil \\ Professora Adjunta de Odontopediatria, Universidade Federal de Pernambuco (UFPE), 50670-901, Recife, PE, Brasil
}

\section{Resumo}

Introdução: A ansiedade odontológica interfere nos cuidados com a saúde bucal, causa resistência em ir ao consultório odontológico e dificulta o trabalho do cirurgião-dentista. Objetivo: determinar a prevalência de ansiedade relacionada ao tratamento odontológico em adolescentes, por meio de uma revisão da literatura. Material e método: Revisão da literatura, realizada através da busca ativa de informações na "Biblioteca Virtual em Saúde" (bvsalud.org) nas bases de dados MEDLINE e LILACS, totalizando a produção bibliográfica de um período de 25 anos. Foram adotados os seguintes descritores: "ansiedade ao tratamento odontológico" e "prevalência", de forma integrada. Foram incluídos estudos originais que avaliaram prevalência de ansiedade ao tratamento odontológico, conduzidos com indivíduos entre 10 e 19 anos de idade, com amostra representativa da população estudada. A busca foi limitada a publicações nos idiomas inglês, português ou espanhol; e do tipo artigo científico. A metodologia seguiu as recomendações PRISMA. Resultados: Foram identificados 7 artigos preenchendo os critérios de inclusão. A prevalência de ansiedade relacionada ao tratamento odontológico variou de 6,5 a $25,6 \%$ entre adolescentes, distribuídos entre Europa, Ásia e Oceania. Houve também diversidade de instrumentos utilizados para avaliação da ansiedade. Conclusão: A prevalência de ansiedade relacionada ao tratamento odontológico apresentou grande variabilidade entre as pesquisas analisadas, podendo chegar a $1 \frac{1}{4}$ dos adolescentes. Observou-se uma carência de estudos de prevalência consistentes nessa faixa etária.

Descritores: Ansiedade ao Tratamento Odontológico; Prevalência; Adolescente.

\section{Abstract}

Introduction: Dental anxiety interferes with oral health care, causes resistance to going to the dental office and hinders the work of dentists. Objective: to determine the prevalence of dental anxiety in adolescents, by means of a review of the literature. Material and method: Integrative review of the literature, conducted by active search in the "Virtual Health Library" (bvsalud.org) in the MEDLINE and LILACS databases, retrieving the bibliographic production in a period of 25 years. The following keywords were selected: "dental anxiety" and "prevalence", combined in an integrated manner. Original studies that evaluated the prevalence of dental anxiety were included, conducted in subjects aged between 10 to 19 years, with a representative sample of the studied population. The search was limited to publications in English, Portuguese and Spanish languages and scientific article type. The methodology followed the PRISMA recommendations. Results: Seven articles that fulfilled the inclusion criteria were identified. The prevalence of dental anxiety ranged from 6.5 to $25.6 \%$ among adolescents, which varied across the countries Europe, Asia and Oceania. Several instruments were used to evaluate dental anxiety. Conclusion: The prevalence of dental anxiety presented great variability among the analyzed studies, reaching up to $1 / 4$ of the adolescents. There was a lack of consistent prevalence data in this age group.

Descriptors: Dental Anxiety; Prevalence; Adolescent.

\section{Resumen}

Introducción: La ansiedad odontológica interfiere en los cuidados con la salud bucal, causa resistencia en ir al consultorio odontológico y dificulta el trabajo del cirujano-dentista. Objetivo: determinar la prevalencia de ansiedad relacionada al tratamiento odontológico en adolescentes, por medio de una revisión de la literatura. Material y método: Revisión de la literatura, realizada a través de la búsqueda activa de informaciones en "Biblioteca Virtual en Salud" (bvsalud.org) en las bases de datos MEDLINE y LILACS, que totalizan la producción bibliográfica de un período de 25 años. Se adoptaron los siguientes descriptores: "ansiedad al tratamiento odontológico" y "prevalencia", de forma integrada. Se incluyeron estudios originales que evaluaron prevalencia de ansiedad al tratamiento odontológico, conducidos con individuos entre 10 y 19 años de edad, con muestra representativa de la población estudiada. La búsqueda se limitó a las publicaciones en Inglés, portugués y español; del tipo artículo científico. La metodología siguió las recomendaciones PRISMA. Resultados: Se identificaron 7 artículos que cumplieron con los criterios de inclusión. La prevalencia de ansiedad relacionada al tratamiento odontológico varía de 6,5 a 25,6\% entre adolescentes, distribuidos entre Europa, Asia y Oceanía. Varios instrumentos fueron utilizados para evaluar la ansiedad. Conclusión: La prevalencia de ansiedad relacionada al tratamiento odontológico presentó gran variabilidad entre las investigaciones analizadas, pudiendo llegar a $1 / 4$ de los adolescentes. Se observó una carencia de datos de prevalencia consistentes en este grupo de edad.

Descriptores: Ansiedad al Tratamiento Odontológico; Prevalencia; Adolescente.

\section{INTRODUÇÃO}

A ansiedade é considerada um estado emocional, momentâneo na maioria dos casos, que o indivíduo experimenta quando se julga vulnerável a algum tipo de perigo, podendo ter ou não fundamento ${ }^{1}$. Na odontologia, a ansiedade tem etiologia multifatorial ${ }^{2}$, e os fatores mais comumente associados são as experiências anteriores dolorosas ${ }^{3,4}$, e a influência de pais que apresentam ansiedade relacionada ao tratamento odontológico ${ }^{3-6}$.

$\mathrm{O}$ medo de ir ao dentista interfere nos cuidados com a saúde bucal, pois pode causar uma resistência em ir ao consultório odontológico, dificultando o andamento do tratamento. Com isso, a ansiedade ao tratamento odontológico pode se relacionar com uma maior experiência de problemas bucais e comportamentais durante o atendimento ${ }^{2,7,8}$.

Pessoas de todas as idades podem ser afetadas pela ansiedade relacionada ao tratamento odontológico, mas seu desenvolvimento ocorre principalmente na infância e na adolescência ${ }^{9}$. De 
acordo com a Organização Mundial de Saúde (OMS) a adolescência delimita-se cronologicamente dos 10 aos 19 anos de idade, e é um período de intensas modificações na vida dos indivíduos, sendo influenciado por processos biológicos, características culturais, condição socioeconômicas, valores e experiências ${ }^{10}$.

A prevalência de ansiedade relacionada ao tratamento odontológico tem sido bastante estudada, devido ao seu impacto na saúde bucal dos indivíduos e a sua influência no trabalho do cirurgião-dentista. No entanto, as estimativas dessa prevalência podem variar consideravelmente a depender de fatores culturais, populacionais e metodológicos ${ }^{11-13}$. Além disso, observa-se que alguns grupos etários tem sido menos investigados, como por exemplo, adolescentes. Sendo assim, este estudo teve como objetivo determinar a prevalência de ansiedade relacionada ao tratamento odontológico em adolescentes, por meio de uma revisão da literatura.

\section{MATERIAL E MÉTODO}

\section{- Estratégia de busca}

A revisão de literatura foi realizada a partir da busca ativa de informações na Biblioteca Virtual de Saúde (BVS - bvsalud.org) nas bases de dados LILACS e MEDLINE. Para tanto, foram adotados os seguintes descritores: "ansiedade ao tratamento odontológico" e "prevalência" (DeCs/MeSH), combinados pelo operador booleano AND. O processo de busca e análise foi conduzido por dois pesquisadores e as publicações foram inseridas no software Mendeley para gerenciar as referências. A última consulta às publicações foi realizada em novembro de 2017.

\section{- Critérios de elegibilidade dos artigos}

Foram incluídos estudos originais que tenham avaliado a prevalência de ansiedade relacionada ao tratamento odontológico em adolescentes, considerando os sujeitos com idades entre 10 e 19 anos, com amostra representativa da população estudada. A busca foi limitada a publicações nos idiomas inglês, português ou espanhol; e do tipo artigo científico. Não houve limite de tempo de publicação. Revisões, metanálises e relatos de caso foram excluídos.

\section{- Seleção das publicações e extração dos dados}

$\mathrm{O}$ processo de análise para avaliação e seleção dos artigos foi realizado por dois pesquisadores, de forma independente, com posterior confronto dos resultados para obtenção dos textos selecionados por consenso. Em casos de divergências ou dúvidas quanto à inclusão dos artigos, houve a participação de um terceiro pesquisador avaliador. Os artigos indexados repetidamente nos dois bancos de dados foram considerados apenas uma vez. Então a seleção das publicações foi conduzida em duas fases: (1) seleção pela leitura dos resumos, e (2) análise qualitativa dos textos na íntegra (Figura 1). Esta metodologia seguiu as recomendações PRISMA (Principais Itens para Relatar Revisões Sistemáticas e Metanálises $)^{14}$.

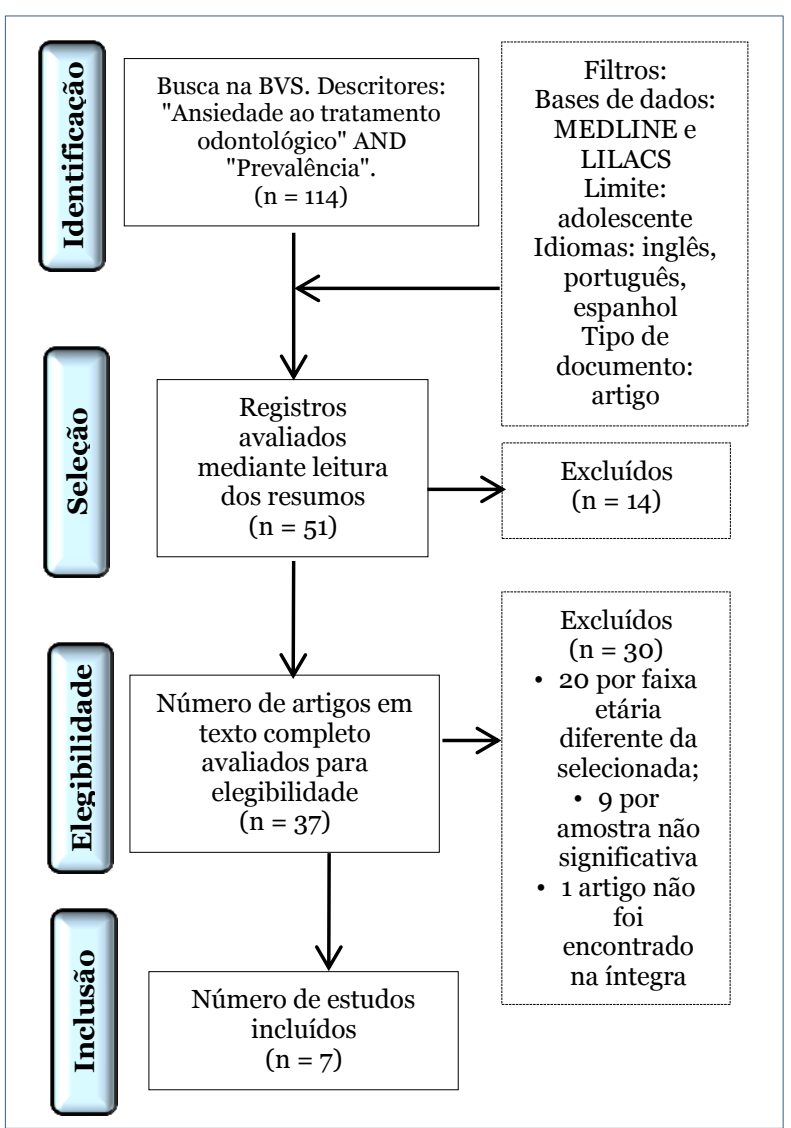

Figura 1: Fluxograma da estratégia de busca e seleção dos artigos via BVS.

\section{RESULTADOS}

Combinando-se os métodos de busca estabelecidos, foram identificados 7 artigos preenchendo os critérios de inclusão (Quadro 1), representando a produção bibliográfica dos últimos 25 anos.

Quadro 1. Prevalência de ansiedade ao tratamento odontológico entre adolescentes

\begin{tabular}{|c|c|c|c|c|c|c|}
\hline $\begin{array}{c}\text { Autor/ } \\
\text { Ano }\end{array}$ & País & $\mathbf{n}$ & Idade & IU & $\%$ & Escore \\
\hline \multirow{2}{*}{$\begin{array}{l}\text { Skaret et } \\
\text { al.3 (1998) }\end{array}$} & \multirow{2}{*}{ Noruega } & \multirow{2}{*}{571} & \multirow{2}{*}{18} & DFS & 19 & $\geq 60$ \\
\hline & & & & DBS & 15 & $>47$ \\
\hline $\begin{array}{c}\text { Murthy et } \\
\text { al. }^{8} \\
(2014)\end{array}$ & Índia & 1.452 & $12-15$ & DAQ & 25,6 & $\begin{array}{c}\text { Respostas } \\
\text { Sim, tenho } \\
\text { medo } \\
\text { Sim, muito } \\
\text { medo }\end{array}$ \\
\hline $\begin{array}{l}\text { Locker et } \\
\text { al. }{ }^{15}(2001)\end{array}$ & $\begin{array}{l}\text { Nova } \\
\text { Zelândia }\end{array}$ & 678 & 15 e 18 & DAS & $\begin{array}{c}15 \text { anos } \\
=10,6 \\
18 \text { anos } \\
=13,3\end{array}$ & $\geq 13$ \\
\hline $\begin{array}{c}\text { Armfield et } \\
\text { al. }{ }^{16} \\
(2006)\end{array}$ & Austrália & 516 & $13^{-17}$ & DAQ & 9,5 & $\begin{array}{c}\text { Respostas } \\
\text { Sim, tenho } \\
\text { medo } \\
\text { Sim, muito } \\
\text { medo }\end{array}$ \\
\hline $\begin{array}{l}\text { Taani }^{17} \\
(2002) \\
\end{array}$ & Jordânia & 1.021 & $12-15$ & DFS & 10 & $\geq 60$ \\
\hline $\begin{array}{l}\text { Stenebrand } \\
\text { et al. }{ }^{18} \\
(2013)\end{array}$ & Suécia & 216 & 15 & DFS & 6,5 & $\geq 60$ \\
\hline $\begin{array}{c}\text { Bedi } \\
\text { et al.19 } \\
(1992)\end{array}$ & Escócia & 1076 & 13 e 14 & DAS & 7,1 & $\geq 15$ \\
\hline
\end{tabular}


A prevalência de ansiedade relacionada ao tratamento odontológico variou de 6,5 a $25,6 \%$ entre adolescentes. Verificou-se também que houve diversidade de instrumentos utilizados para avaliação da ansiedade.

Considerando-se o país de coleta de dados, pode-se observar a escassez de estudos de prevalência de ansiedade relacionada ao tratamento odontológico em adolescentes. Sendo que os resultados deste estudo encontram-se distribuídos entre Europa, Ásia e Oceania (Figura 2).

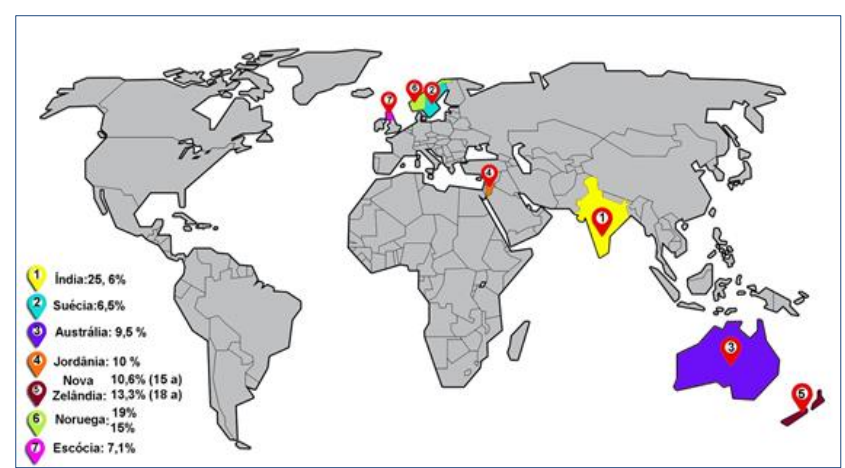

Figura 2: Distribuição geográfica dos estudos incluídos e prevalência de ansiedade ao tratamento odontológico entre adolescentes.

\section{DISCUSSÃO}

Em relação à fonte de informação para a coleta de dados, todos os estudos selecionados utilizaram dados primários e apenas dois não foram realizados em ambiente escolar. Destes, um foi parte de uma coorte de nascidos vivos ${ }^{15}$ e o outro teve os dados coletados mediante entrevistas telefônicas ${ }^{16}$.

As pesquisas avaliaram a ansiedade ao tratamento odontológico por meio de quatro diferentes instrumentos, mas todos deram ênfase aos resultados sobre ansiedade elevada (high dental anxiety). Quando o DAQ foi empregado, considerouse ansiedade elevada às respostas "Sim, tenho medo" e "Sim, muito medo"8,16. Para o DFS foi necessário ter um escore maior ou igual a $60^{3,17,18}$. Enquanto que para o DBS considerou-se um escore maior ou igual a quarenta e oito ${ }^{3}$. Em relação ao DAS ocorreu divergência entre os parâmetros adotados pelos autores para a classificação de ansiedade elevada. No estudo realizado na Escócia levou-se em consideração DAS maior ou igual a quinze ${ }^{19}$, já no estudo da Nova Zelândia definiu-se ansiedade elevada por DAS maior ou igual a treze ${ }^{15}$. Esse fator pode ter influenciado na diferença da prevalência observada nesses dois estudos. Onde no primeiro, aos 13 e 14 anos de idade, a prevalência de ansiedade elevada foi de $7,1 \%$, enquanto que no segundo a prevalência aos 15 anos foi de $10,6 \%{ }^{15,19}$.

Confrontando-se os resultados dos estudos que avaliaram as mesmas idades, observa-se que aos 15 anos os adolescentes que apresentaram elevada ansiedade ao tratamento odontológico corresponderam a $6,5 \%$ na Suécia ${ }^{18}$ e $10,6 \%$ na Nova
Zelândia ${ }^{15}$. Já aos 18 anos, na Noruega, a prevalência foi de $15 \%$ por meio do DBS e de e $19 \%$ pelo DFS ${ }^{3}$, havendo correlação significativa entre esses dois instrumentos. Enquanto que na Nova Zelândia a prevalência foi igual a $13,3 \%{ }^{15}$.

Ainda nesse estudo neozelandês verificou-se que, quando avaliados individualmente, $10,1 \%$ dos adolescentes que não eram ansiosos aos 15 anos tornaram-se ansiosos aos 18, o que demonstra que esse tipo de ansiedade não surge exclusivamente na infância. Por outro lado, a maioria dos adolescentes que apresentaram ansiedade aos 15 anos não continuaram ansiosos aos 18 anos. Assim, os autores sugerem que a ansiedade ao tratamento odontológico está sujeita a altas taxas de remissão ${ }^{15}$. Em contrapartida, em um estudo transversal realizado na Austrália com indivíduos de diferentes idades (inclusive adolescentes), constatou-se que a prevalência de ansiedade ao tratamento odontológico sofre um aumento crescente nas faixas etárias seguintes à adolescência. Os autores explicam essa diferença devido às mudanças biológicas, culturais e sociais que ocorrem ao longo da vida humana ${ }^{16}$.

Com relação à existência ou não de diferença no padrão de ansiedade relacionada ao tratamento odontológico entre os gêneros, os achados dos estudos são divergentes. Os resultados de Skaret et al. ${ }^{3}$ mediante o emprego do DFS, mostraram diferença significante entre os gêneros, sendo a prevalência maior no gênero feminino, corroborando com os resultados de outras pesquisas ${ }^{18,19}$. No entanto, no mesmo estudo ${ }^{3}$, quando foi utilizado o instrumento DBS não foi verificada diferença entre os gêneros. Da mesma forma, Locker et al. ${ }^{15}$ não encontraram diferença na prevalência de ansiedade quanto ao gênero em nenhuma das idades avaliadas.

A ansiedade pode ser uma faceta da personalidade de indivíduos que apresentam outras fobias, além da relacionada ao tratamento odontológico $^{3}$. Isso foi verificado em dois estudos ${ }^{3,19}$ os quais, por meio do instrumento GFS (Geer Fear Scale), observaram que a ansiedade ao tratamento odontológico esteve fortemente relacionada à ansiedade fóbica de um modo geral. Essas mesmas pesquisas apontam ainda que a ansiedade ao tratamento odontológico em adolescentes é influenciada por pessoas próximas a estes que também apresentam esse tipo de ansiedade, sendo isso considerado um fator preditivo de ansiedade ao tratamento odontológico nessa faixa etária.

Outro fator preditivo de ansiedade ao tratamento odontológico foi à ocorrência de dor na última consulta odontológica ou experiência prévia de dor em tratamento odontológico ${ }^{3,18}$. De acordo com os resultados de Skaret et al. ${ }^{3}$, os adolescentes que relataram mais de uma experiência dolorosa ou sessão de tratamento odontológico desagradável tiveram 9,9 vezes mais chances de ter elevada 
ansiedade relacionada ao tratamento odontológico.

Além disso, adolescentes com elevada ansiedade ao tratamento odontológico mostraram-se mais propensos a ter cárie dentária não tratada, sofrendo as consequências clínicas, como comprometimento pulpar. Esse efeito pode ser explicado pelo ciclo vicioso, em que a ansiedade faz o indivíduo evitar a visita ao dentista, levando a atrasos no recebimento de cuidados ou tratamento preventivo adequado ${ }^{8}$.

Nesse sentido, quanto maior o tempo desde a última visita ao dentista, maior o grau de ansiedade ao tratamento odontológico, sendo esse outro fator preditivo desse tipo de ansiedade em adolescentes ${ }^{19}$. Porém, para Skaret et al. ${ }^{3}$ houve uma correlação muito fraca, mas estatisticamente significativa, entre a ansiedade ao tratamento odontológico e o tempo desde a última consulta odontológica do adolescente. $\mathrm{E}$, em uma pesquisa realizada na Jordâni ${ }^{17}$ a maioria dos adolescentes relatou visitar o dentista apenas em casos de urgência.

$\mathrm{O}$ maior percentual de ansiedade foi observado na Índia, onde 25,6\% dos escolares de 12 a 15 anos avaliados foram considerados com elevada ansiedade ao tratamento odontológico ${ }^{8}$. Dentre os países onde foram realizados os sete estudos compreendidos nesta revisão, a Índia é o que apresenta o menor Índice de Desenvolvimento Humano (IDH). O país ocupa o centésimo trigésimo lugar com um IDH de 0,609, sendo considerado um país de médio desenvolvimento humano. Em contrapartida, Noruega e Austrália são os dois primeiros do Ranking, com IDH de 0,944 e 0,935 respectivamente $^{20}$.

Destaca-se que a prevalência de ansiedade relacionada ao tratamento odontológico foi elevada na Noruega, apesar de elevado IDH. No entanto, o estudo realizado com adolescentes noruegueses foi publicado em 1998, enquanto o estudo realizado na Índia foi mais recente, publicado em 2014.

Assim, o nível socioeconômico parece estar associado à prevalência de elevada ansiedade ao tratamento odontológico. Isso foi verificado em uma pesquisa realizada com adolescentes escoceses de 13 e 14 anos, na qual os grupos de classe social mais baixa apresentam maior ansiedade ${ }^{19}$. Nesse sentido, em um estudo com 1.021 adolescentes de 12 a 15 anos observou-se uma maior prevalência desse tipo de ansiedade nos estudantes das escolas públicas (12\%) quando comparados aos de escolas privadas $(7 \%)^{17}$. Mas independente do nível socioeconômico, em ambas as pesquisas, os procedimentos relacionados com o uso de agulha e broca eram o que causavam maior medo ${ }^{17,19}$.

Dessa forma, levando-se em consideração a prevalência de ansiedade relacionada ao tratamento odontológico em adolescentes e seu impacto na saúde bucal, existem claras implicações clínicas tanto para o paciente, como para os profissionais de odontologia. Assim, é importante que os cirurgiõesdentistas e auxiliares sejam capazes de identificar pacientes ansiosos para tratá-los adequadamente, fornecendo mais informações, fazendo pausas durante o procedimento, e utilizando técnicas de distração e anestesia mais eficiente ${ }^{16}$.

\section{CONCLUSÃO}

A prevalência de ansiedade relacionada ao tratamento odontológico apresentou grande variabilidade entre as pesquisas analisadas, podendo chegar a um quarto dos adolescentes. Observou-se uma carência de estudos de prevalência consistentes nessa faixa etária.

\section{AGRADECIMENTOS}

À Coordenação de Aperfeiçoamento de Pessoal de Nível Superior - CAPES. As autoras Trícia Murielly Andrade de Souza Mayer, Patrícia Diletieri Assis, Kamila Azoubel Barreto e Carolina Maria Maciel Pessoa da Silva receberam suporte financeiro da Coordenação de Aperfeiçoamento de Pessoal de Nível Superior (CAPES) em formato de bolsa de estudos e pesquisas.

\section{REFERÊNCIAS}

1. Oliveira MLRS, Araújo SM, Bottan ER. Ansiedade ao tratamento odontológico: perfil de um grupo de adultos em situação não clínica. Arq Ciênc Saúde UNIPAR. 2016;19(3):165-70.

2. Oliveira MMT, Colares V. The relationship between dental anxiety and dental pain in children aged 18 to 59 months: a study in Recife, Pernambuco State, Brazil. Cad Saúde Pública. 2009;25(4):743-50.

3. Skaret E, Raadal M, Berg E, Kvale G. Dental anxiety and dental avoidance among 12 to 18 year olds in Norway. Eur J Oral Sci. 1999; 107(6):422-28.

4. Ferreira AMB, Colares V. Validação da Versão Brasileira Curta do Fear of Dental Pain Questionnaire - Short Form (S-FDPQ). Pesqui bras odontopediatria clin integr. 2011;11(2):275-79.

5. Klingberg G. Dental fear and behaviour management problems in children. A study of measurement, prevalence, concomitant factors, and clinical effects. Swed Dent J Suppl. 1995; 103:1-78.

6. Soares FC, Lima RA, Barros MVG, Dahllöf G, Colares V. Development of dental anxiety in schoolchildren: A 2-year prospective study. Community Dent Oral Epidemiol. 2017; 45(3):281-88.

7. Caraciolo G, Colares V. Prevalência de medo e/ou ansiedade relacionados à visita ao dentista em crianças com 5 anos de idade na cidade do Recife. Rev odonto ciênc. 2004;19(46):348-53.

8. Murthy AK, Pramila M, Ranganath S. Prevalence 
of clinical consequences of untreated dental caries and its relation to dental fear among 12-15-yearold schoolchildren in Bangalore city, India. Eur Arch Paediatr Dent. 2014;15(1):45-9.

9. Assuncão CM, Losso EM, Andreatini R, Menezes JV. The relationship between dental anxiety in children, adolescents and their parents at dental environment. J Indian Soc Pedod Prev Dent. 2013;31(3):175-79.

10.World Health Organization. Young People's Health - a Challenge for Society. Report of a WHO Study Group on Young People and Health for All. Technical Report Series 731. Geneva: WHO; 1986.

11.Klingberg G, Broberg AG. Dental fear/anxiety and dental behaviour management problems in children and adolescents: a review of prevalence and concomitant psychological factors. Int $\mathbf{J}$ Paediatr Dent. 2007;17(6):391-406.

12.Beena JP. Dental subscale of children's fear survey schedule and dental caries prevalence. Eur J Dent. 2013;7(2):181-85.

13. Barreto KA, Lima DSM, Soares FC, Colares V. Ansiedade relacionada ao tratamento odontológico em pacientes infantis: uma revisão da literatura. Odontol Clín-Cient. 2016;15(1):7-11.

14. Moher D, Liberati A, Tetzlaff J, Altman DG. The PRISMA Group. Preferred Reporting Items for Systematic Reviews and Meta-Analyses: The PRISMA Statement. PLoS Med. 2009; 6(7):e1000097

15. Locker D, Thomson WM, Poulton R. Onset of and patterns of change in dental anxiety in adolescence and early adulthood: a birth cohort study. Community Dent Health. 2001;18(2):99-104.

16. Armfield JM, Spencer AJ, Stewart JF. Dental fear in Australia: who's afraid of the dentist? Aust Dent J. 2006;51(1):78-85.

17. Taani DQ. Dental attendance and anxiety among public and private school children in Jordan. Int Dent J. 2002;52(1):25-9.

18. Stenebrand A, Boman UW, Hakeberg M. Dental anxiety and temperament in 15-year olds. Acta Odontol Scand. 2013;71:15-21.

19. Bedi R, Sutcliffe P, Donnanz PT, Mcconnachie J. The prevalence of dental anxiety in a group of 13and 14-year-old Scottish children. Int J Paediatr Dent.1992; 2(1):17-24.

20. Organização das Nações Unidas. PNUD Programa das Nações Unidas para o Desenvolvimento. Relatório de Desenvolvimento Humano 2015, 2015 [citado 2017 Maio 09] Disponível em: http://www.br.undp.org/ content/ brazil/pt/home/idh0/rankings/idh-global.html.
CONFLITO DE INTERESSES

Os autores declaram não haver conflitos de interesse.

AUTOR PARA CORRESPONDENCIA

Trícia Murielly Andrade de Souza Mayer triciamurielly@yahoo.com.br

Submetido em 01/08/2018

Aceito em 04/10/2018 